\title{
Nanoscale Computer Operates at the Speed of Light
}

\author{
Predictions indicate that a nanometer-sized wave-based computer could \\ solve equations in a fraction of the time of their larger, electronic \\ counterparts.
}

\author{
By Sarah Wells
}

$\square$ ooting up your laptop may seem like an instantaneous process, but in reality, it's an intricate dance of signals being converted from analog wave forms to digital bytes to photons that deliver information to our retinas. For most computer uses, this conversion time has no impact. But for supercomputers crunching reams of data, it can create a serious, energy-consuming slowdown. Researchers are looking to solve this problem using analog, wave-based computers, which operate solely using light waves and can perform calculations faster and with less energy. Now, Heedong Goh and Andrea Alù from the Advanced Science Research Center at the City University of New York present the design for a nanosized wave-based computer that can solve mathematical problems, such as integro-differential equations, at the speed of light [1].

One route that researchers have taken to make wave-based analog computers is to design them into metamaterials, materials engineered to apply mathematical operations to

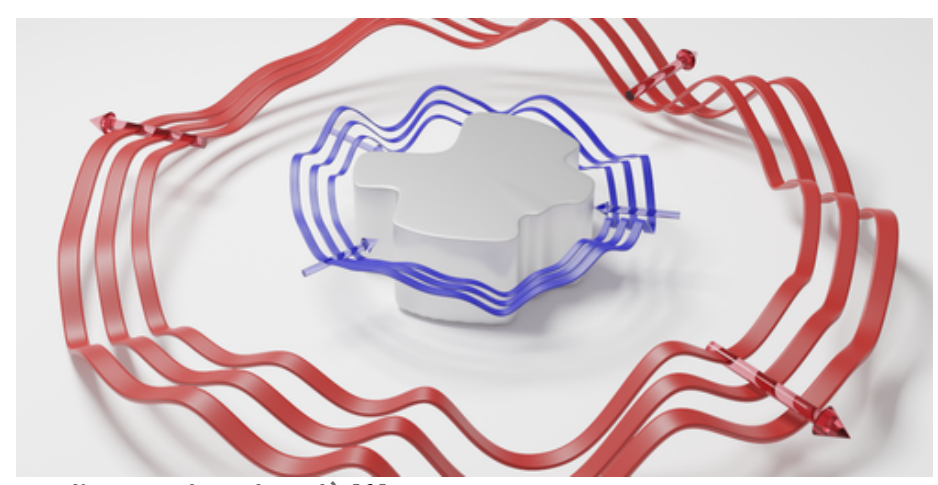

Credit: H. Goh and A. Alù [1] incident light waves. Previous designs used large-area metamaterials-up to two square feet $\left(\sim 0.2 \mathrm{~m}^{2}\right)$-limiting their scalability. Goh and Alù have been able to scale down these structures to the nanoscale, a length scale suited for integration and scalability.

The duo's proposed computer is made from silicon and is crafted in a complex geometrical nanoshape that is optimized for a given problem. Light is shone onto the computer, encoding the input, and the computer then encodes the solution to the problem onto the light it scatters. For example, the duo finds that a warped-trefoil structure can provide solutions to an integral equation known as the Fredholm equation.

Goh and Alù's calculations indicate that their nanosized wave-based computers should be able to solve problems with near-zero processing delay and with negligible energy consumption.

Sarah Wells is a freelance science journalist based in Boston.

\section{REFERENCES}

1. H. Goh and A. Alù, "Nonlocal scatterer for compact wave-based analog computing," Phys. Rev. Lett. 128, 073201 (2022). 